\title{
Relationship between bile salts, bacterial translocation and duodenal mucosal integrity in functional dyspepsia
}

\section{Bile salts in functional dyspepsia}

Beeckmans Dorien ${ }^{1}$, Farré Ricard ${ }^{1}$, Riethorst Danny², Keita Åsa V³ ${ }^{3}$ Augustijns Patrick², Söderholm Johan $D^{3}$, Vanuytsel Tim ${ }^{1}$,Vanheel Hanne ${ }^{1}$, Tack Jan ${ }^{1}$

${ }^{1}$ Department of chronic diseases, metabolism and ageing (ChroMeTa), Translational Research Center for Gastrointestinal Disorders (TARGID), KU Leuven, Leuven, Belgium

${ }^{2}$ Drug Delivery and Disposition, Department of Pharmaceutical and Pharmacological Sciences, KU Leuven, Leuven, Belgium

${ }^{3}$ Department of Clinical and Experimental Medicine, Linköping University, Linköping, Sweden

Correspondence Jan Tack, Department of chronic diseases, metabolism and ageing, Translational Research Center for Gastrointestinal Disorders, KU Leuven Campus Gasthuisberg, O\&N1 Herestraat 49 - Box 701, $7^{\text {th }}$ floor, 3000 Leuven, Belgium. E-mail: jan.tack@uzleuven.be.

Fax: +32 3444 19. Telephone: +32 16377474 . 


\begin{abstract}
Background Functional dyspepsia is a complex disorder, in which multiple mechanisms underlie symptom generation, including impaired duodenal barrier function. Moreover, an altered duodenal bile salt pool was recently discovered in patients with functional dyspepsia. We aimed to evaluate the relationship between bile salts, bacterial translocation and duodenal mucosal permeability in functional dyspepsia.
\end{abstract}

Methods Duodenal biopsies from patients with functional dyspepsia and healthy volunteers were mounted in Ussing chambers to measure mucosal resistance and bacterial passage in the absence and presence of fluorescein conjugated Escherichia coli and glyco-ursodeoxycholic acid exposure. In parallel, duodenal fluid aspirates were collected from patients and bile salts were analyzed.

Key results The transepithelial electrical resistance of duodenal biopsies from patients was lower compared to healthy volunteers $\left(21.4 \pm 1.3 \Omega . \mathrm{cm}^{2}\right.$ vs. $\left.24.4 \pm 1.2 \Omega . \mathrm{cm}^{2} ; \mathrm{p}=0.02 ; \mathrm{N}=21\right)$. The ratio of glycocholic and glyco-chenodeoxycholic acid to tauro- and glyco-ursodeoxycholic acid correlated positively with TEER in patients. Glyco-ursodeoxycholic acid slightly altered the mucosal resistance, resulting in similar values between patient and healthy biopsies $\left(22.1 \pm 1.0 \Omega . \mathrm{cm}^{2}\right.$ vs. $\left.23.0 \pm 1.0 \Omega . \mathrm{cm}^{2} ; \mathrm{p}=0.5\right)$. Bacterial passage after 120 minutes was lower for patient than for healthy biopsies $(0.0(0.0-681.8) \mathrm{vs}$. 1684.0 (0.0-4773.0) E. coli units; p=0.02). Glyco-ursodeoxycholic acid increased bacterial passage in patient biopsies (102.1 (0.0-733.0) vs. 638.9 (280.6-2124.0) E. coli units; p=0.009). No correlation was found between mucosal resistance and bacterial passage.

Conclusions \& inferences Patients with functional dyspepsia displayed decreased duodenal mucosal resistance associated with bile salts, however not associated with bacterial passage in vitro. In addition, the hydrophilic bile salt glyco-ursodeoxycholic acid abolished differences in mucosal resistance and bacterial passage between patient and control group.

Key words Bacterial passage \& uptake, bile salts, duodenal permeability, functional dyspepsia. 


\section{INTRODUCTION}

Patients with functional dyspepsia (FD) suffer from recurrent or persistent gastroduodenal symptoms without evidence of a structural disease that is likely to explain the symptoms. ${ }^{1}$ Despite a high prevalence in the population, available treatment options are limited and the exact mechanism behind the pathophysiology of FD is still obscure. ${ }^{2}$ FD is most likely a complex disorder, in which symptom generation depends on multiple mechanisms, including gastric sensorimotor dysfunction, duodenal mucosal low-grade immune activation and impaired duodenal barrier function. ${ }^{3}$

In FD, impaired duodenal barrier function is present as an increased duodenal paracellular permeability and an associated decreased expression of mucosal cell-to-cell adhesion molecules. ${ }^{2}$ It was hypothesized by Vanheel et al. and others that impaired barrier function allows transepithelial passage of luminal antigens, resulting in low-grade inflammation. ${ }^{3}$ However, mucosal permeability may be influenced by multiple factors, including genetic factors, stress and duodenal luminal factors, such as bile salts. ${ }^{3-5}$

Hydrophobic bile salts were shown to impair the small intestinal barrier function and increase the intestinal permeability. ${ }^{6-7}$ An impaired intestinal barrier function may in turn result in bacterial translocation, which is the passage of bacteria and bacterial products across the intestinal barrier, as reported for colonic biopsies of patients with irritable bowel syndrome (IBS) ${ }^{8-9}$ In a study by Münch et al., it was shown that hydrophobic bile salts worsen mucosal barrier dysfunction in colonic biopsies of patients with collagenous colitis in remission, resulting in increased bacterial uptake. ${ }^{10}$

A duodenal altered bile salt composition and a decreased bile salt concentration was recently discovered by our group in patients with FD. ${ }^{11-13}$ We hypothesized that these alterations in the duodenal bile salt pool in FD may contribute to impaired duodenal mucosal integrity, resulting in increased bacterial uptake via the transcellular pathway, eventually leading to low-grade inflammation. Therefore, we aimed to evaluate the relationship between bile salts, bacterial uptake and duodenal mucosal permeability in patients with FD and healthy volunteers (HV). 


\section{MATERIALS AND METHODS}

\section{Study population}

Patients fulfilling Rome IV criteria for FD, who were scheduled for upper gastrointestinal endoscopy with duodenal biopsies, were prospectively recruited from the outpatient clinic of the Department of Gastroenterology at the University Hospitals Leuven. Patients with FD and HV were also recruited via a mailing list. Exclusion criteria were: first-degree relatives with celiac disease, diabetes mellitus, allergy/atopy (eczema, asthma, allergic rhinoconjunctivitis), coagulation disorders/anticoagulant therapy and first-degree relatives with Crohn's disease or type I diabetes mellitus. None of the participants was allowed to take antihistamines, ketotifen, cromoglycate, acetylsalicylates, non-steroidal anti-inflammatory drugs, anticholinergics, theophylline, $\beta 2$-agonists, codeine or opioid derivatives and proton pump inhibitors for at least 2 weeks prior to the gastroscopy. Psychosocial co-morbidity was assessed by the patient health questionnaire-9 (PHQ-9) and the visceral sensitivity index (VSI) questionnaire was used to evaluate gastrointestinal-specific anxiety. The dyspepsia symptom score (DSS) was used to score the dyspeptic symptom intensity over the preceding 3 months in patients with FD and HV. ${ }^{14}$ These protocols were approved by Leuven University Hospitals Ethics Committee (s56910; 18/12/2015 and s56880; 10/1/2017), and all participants approved and signed the informed consent before inclusion in the studies.

\section{Study 1: Duodenal mucosal integrity, fluid aspiration and bile salt analysis}

Upper gastroduodenoscopy was performed by an experienced endoscopist to obtain three biopsy samples from the second part of the duodenum with a standard biopsy forceps (Radial Jaw3, outside diameter $2.2 \mathrm{~mm}$; Boston Scientific, Natick, Massachusetts, USA). ${ }^{3}$ The biopsy specimens were collected in ice-cold oxygenated Krebs-Ringer bicarbonate buffer and mounted in modified Ussing chambers (Mussler Scientific Instruments, Aachen, Germany), with an exposed tissue area of $1.76 \mathrm{~mm}^{2}$, following the same protocol as stated by Vanheel et al. ${ }^{3}$ Mucosal integrity was measured as transepithelial electrical resistance (TEER) and paracellular passage of a $4 \mathrm{kDa}$ fluorescein isothiacyanate-labeled dextran (FITC-dx4). ${ }^{3}$ After the gastroduodenoscopy, duodenal fluids were aspirated via a nasoduodenal catheter at fixed time points before and after a liquid meal 
(FortimelEnergy®; Nutricia, Zoetendaal, The Netherlands, $150 \mathrm{kcal}$ per $100 \mathrm{~mL}$ with $5.9 \mathrm{~g}$ proteins, $18.4 \mathrm{~g}$ carbohydrates and $5.8 \mathrm{~g}$ lipids, pH6.7) and bile salt concentration was analyzed with liquid chromatography-mass spectrometry/mass spectrometry (LC-MS/MS), as previously reported. ${ }^{11,15}$ Bile salts were expressed as absolute concentrations $(\mathrm{mM})$ and total bile salts was the molar sum of all measured bile salts. ${ }^{11}$ Fasting and fed bile salts were calculated as the average of all time points in fasted and fed state. ${ }^{11}$ Primary bile salts were the molar sum of glyco-cholic acid (GCA), tauro-cholic acid (TCA), glyco-chenodeoxycholic acid (GCDCA) and tauro-chenodeoxycholic acid (TCDCA). Secondary bile salts were the molar sum of glyco-deoxycholic acid (GDCA), tauro-deoxycholic acid (TDCA), glyco-ursodeoxycholic acid (GUDCA) and tauro-ursodeoxycholic acid (TUDCA). The bile salt peak occurred approximately 30 minutes after liquid meal intake and was characterized by the maximal amount of total bile salts postprandially.

\section{Study 2: Evaluation of bacterial uptake}

Gastroduodenoscopy was performed to obtain eight duodenal biopsy samples per participant. The biopsies were put in ice-cold oxygenated Krebs-Ringer bicarbonate buffer. The biopsies were mounted in Ussing chambers with an exposed tissue area of $1.76 \mathrm{~mm}^{2}$ using the technique previously described by Wallon et al. ${ }^{16}$ Mucosal and serosal compartments were filled with $3 \mathrm{ml} 10 \mathrm{mM}$ mannitol and 10mM glucose in Krebs-Ringer bicarbonate buffer, respectively. ${ }^{3}$ Solutions were kept at $37^{\circ} \mathrm{C}, \mathrm{pH} 7.4$ and continuously carbonated with $\mathrm{O} 2 / \mathrm{CO} 2(95 / 5 \%) .{ }^{3}$ Experiments were performed in open-circuit conditions and started with a 30-min equilibration period to establish a steady state. ${ }^{3,16}$ TEER was measured for $120 \mathrm{~min}$ in all biopsies. ${ }^{16}$ After equilibration, chemically killed fluorescein-conjugated Escherichia coli K-12 BioParticles (Molecular Probes, Leiden, the Netherlands) were added to the mucosal side at a final concentration of $2.10^{6}$ E. coli units $/ \mathrm{ml} .{ }^{17} \mathrm{We}$ have already published that patients with FD have an increased permeability to ions as shown by a reduced TEER and that they have an increased paracellular permeability to FD4. In the present study, we wanted to explore whether the transcellular instead of the paracellular pathway is affected. Larger molecules of $40 \mathrm{KDa}$ and bacteria cross the intestinal epithelium via the transcellular pathway. We selected a fluorescein-conjugated E. coli to assess the transcellular uptake. These bacteria were killed with paraformaldehyde which stops 
their reproduction but retains antigenicity. ${ }^{17}$ Half of the biopsies per participant were also exposed to 1 , 2 or 5mM GUDCA ( $\mathrm{pH} 7.8 \pm 0.3$; MW 449.62; Sigma-Aldrich BVBA, Overijse, Belgium) to evaluate the influence of GUDCA on TEER and bacterial uptake in duodenal biopsies of patients with FD and HV. GUDCA, was diluted in Krebs containing $10 \mathrm{mM}$ of mannitol to obtain concentrations of $1 \mathrm{mM}, 2 \mathrm{mM}$ and $5 \mathrm{mM} \cdot{ }^{10}$ We analyzed the data concerning the separate $(1,2$ and $5 \mathrm{mM})$ and the pooled GUDCA conditions (all data together). At time point 0 and after 120 minutes, $300 \mu \mathrm{L}$ of the serosal compartments were collected and analyzed in a fluorescence reader (FLUOstar Omega; BMG Labech, Ortenberg, Germany) at $480 \mathrm{~nm}$ excitation and $520 \mathrm{~nm}$ emission level. ${ }^{17}$ We calculated the E. coli units from fluorescence intensity using an E. coli standard curve. In addition, two biopsies per participant (one exposed to $E$. coli and one exposed to $E$. coli and a certain concentration of GUDCA) were fixated with $4 \%$ formaldehyde in phosphate buffered saline (PBS) (pH 7.4) at time points 30,45 or 120 minutes at $4^{\circ} \mathrm{C}$ overnight. Afterwards, the biopsies were kept in $30 \%$ sucrose for at least 2 days. After fixation, the biopsies were positioned in a cryomold with tissue tek O.C.T. compound (Sakura Finetek Belgium BVBA) and stored at $-80^{\circ} \mathrm{C}$ until sectioning using a cryostat microtome. Sections of $10 \mu \mathrm{m}$ dried overnight at room temperature. ${ }^{10}$ Prolong Gold antifade with DAPI was used as mounting medium for nuclear and counterstaining. Images were obtained using an XM10 fluorescence microscope (Olympus, Tokyo, Japan) at 40x magnification.

\section{Statistical analysis}

Data were analyzed using GraphPad prism 7 and statistical significance was set at $p<0.05$. Two-tailed paired t-tests or Wilcoxon signed rank tests, depending on data distribution, were performed to analyze between-group differences. Pearson or spearman correlation coefficient was used, where appropriate, for correlation analysis. Finally, correction for multiple testing (Bonferroni) was performed. Parametric values were presented as mean $\pm \mathrm{SEM}$, while non-parametric values were presented as median $(25-75$ th interquartile range). 


\section{RESULTS}

Study 1: Duodenal mucosal integrity, fluid aspiration and bile salt analysis

\section{Study population}

Three duodenal biopsies and aspirates were obtained from 18 patients with FD (35.4 \pm 3.3 years, 6 men, Table 1). Table 2 summarizes the grading of dyspeptic symptoms. Two patients were subtyped as EPS, seven as PDS and nine belonged to the EPS/PDS overlap group based on Rome IV criteria. ${ }^{1}$ Based on previously published criteria, two patients were assumed to have post-infectious FD; 13 patients fulfilled criteria for co-morbid IBS. ${ }^{18}$

\section{Correlation between bile salts and duodenal permeability in patients with functional dyspepsia}

This patient group showed an average TEER of $20.7 \pm 1.2 \Omega . \mathrm{cm}^{2}$ and permeability for FITC-dx4 of $35.8 \pm 4.1 \mathrm{pmol}$. TEER and paracellular passage were negatively correlated $(\mathrm{r}=-0.82 ; \mathrm{p}<0.0001)$. We evaluated possible correlations between individual bile salts, total bile salts, primary bile salts and secondary bile salts concentrations and paracellular passage and TEER (Table 3). In patients with FD, a positive correlation was found between the ratio of GCA and GCDCA to TUDCA and GUDCA and TEER in the fasted $(\mathrm{r}=0.56 ; \mathrm{p}=0.03 ; \mathrm{N}=15)$ and the fed state $(\mathrm{r}=0.61 ; \mathrm{p}=0.02 ; \mathrm{N}=14)$. In addition, a positive correlation was found between secondary bile salts and paracellular passage $(r=0.60 ; p=0.03$, $\mathrm{N}=13$ ) during the bile salt peak. However, none of these correlations persisted after Bonferroni correction for multiple testing. Duodenal aspirate data were obtained from 17 patients in the fasting state and from 15 patients in the fed state due to incorrect positioning of the catheter in the duodenum. The different number of patients between the correlation with TEER and with paracellular passage is due to methodological problems when assessing FD4-Dextran permeability in two participants.

\section{Study 2: Evaluation of bacterial uptake and in vitro effect of GUDCA}

\section{Study population}

Eight duodenal biopsies were obtained from 21 patients with FD (42.7 \pm 3.2 years, 6 men) and $21 \mathrm{HV}$ (38.8 \pm 2.9 years, 6 men) (Table 4). Table 5 summarizes the grading of dyspeptic symptoms. Three patients were subtyped as EPS, eight as PDS and nine belonged to the EPS/PDS overlap group based on 
Rome IV criteria. ${ }^{1}$ Based on previously published criteria, four patients were assumed to have postinfectious FD; 16 patients fulfilled criteria for co-morbid IBS. ${ }^{18}$

\section{Duodenal mucosal epithelial resistance and $E$. coli passage}

The duodenal mucosal TEER in biopsies was significantly lower in patients with FD compared to HV $\left(21.4 \pm 1.3 \Omega . \mathrm{cm}^{2}\right.$ vs. $24.4 \pm 1.2 \Omega . \mathrm{cm}^{2}, \mathrm{p}=0.02, \mathrm{~N}=21$ for each group; Figure $\left.1 \mathrm{~A}\right)$. The number of $E$. coli units measured at the basolateral side after 120 minutes was lower in patient biopsies than in HV biopsies (0.0 (0.0-681.8) vs. $1684.0(0.0-4773.0)$ E. coli units; $\mathrm{p}=0.02, \mathrm{~N}=21$ for each group; Figure 1B).

Glyco-ursodeoxycholic acid abolished the differences in epithelial resistance and E. coli passage between patient and control group

Exposure of duodenal biopsies from patients and HV to GUDCA did not significantly alter the TEER $\left(22.1 \pm 1.0 \Omega . \mathrm{cm}^{2}\right.$ vs. $21.4 \pm 1.3 \Omega . \mathrm{cm}^{2}, \mathrm{p}=0.5$ and $23.0 \pm 1.0 \Omega . \mathrm{cm}^{2}$ vs. $24.4 \pm 1.2 \Omega . \mathrm{cm}^{2}, \mathrm{p}=0.1$, respectively; Figure 1A). However, after exposure to GUDCA, the TEER values between patient and HV biopsies were similar $(\mathrm{p}=0.5$ ). In addition, TEER of patient biopsies exposed to GUDCA was similar to TEER of HV biopsies in standard conditions ( $\mathrm{p}=0.2)$. Furthermore, GUDCA exposure increased E. coli passage in patient biopsies (102.1 (0.0-733.0) vs. 638.9 (280.6-2124.0) E. coli units; p=0.009), while, GUDCA exposure had no effect on E. coli passage in HV biopsies (1066.0 (0.0-4651.0) vs. 459.2 (0.0-2100.0) E. coli units; p=0.74) (Figure 1B). Patient and HV biopsies with GUDCA exposure showed similar $E$. coli passage after 120 minutes (708.3 (344.4-1550) vs. 369.9 (0-2100) E. coli units; p=0.28). In addition, E. coli passage in patient biopsies exposed to GUDCA was similar to E. coli passage in HV biopsies at baseline ( $\mathrm{p}=0.7$ ). Fluorescence microscopy was used to confirm E. coli uptake in the epithelial cell layer of patient and HV duodenal mucosal biopsies after 120 minutes exposure to fluorescent bacteria with and without exposure to GUDCA. 
Exposure to glyco-ursodeoxycholic acid decreased E. coli passage in duodenal biopsies of healthy volunteers

Patient and HV biopsies were exposed to $1 \mathrm{mM}$ GUDCA ( $\mathrm{N}=11$ for each group), $2 \mathrm{mM}$ GUDCA ( $\mathrm{N}=5$ for each group) or 5mM GUDCA ( $\mathrm{N}=5$ for each group) compared to no GUDCA exposure ( $\mathrm{N}=21$ for each group). No concentration-dependent effect of GUDCA was noticed on TEER and E. coli passage in patient biopsies (One-way ANOVA $\mathrm{P}=0.2$ and $\mathrm{P}=0.23$, respectively; Figure 2A, 2C).

However, E. coli passage was decreased in HV biopsies after exposure to $1 \mathrm{mM}$ GUDCA compared to standard conditions (76.5 (0.0-550.6) vs. 1035.0 (0.0-4617.0) E. coli units; $\mathrm{p}=0.03$; Figure 2D).

\section{Correlation between duodenal permeability and bacterial passage}

Correlation analysis showed only a strong correlation between TEER and E. coli passage in duodenal biopsies of $\mathrm{HV}$ after exposure to $2 \mathrm{mM}$ GUDCA ( $\mathrm{r}=0.99 ; \mathrm{p}=0.01$; $\mathrm{N}=4$ ). However, the sample size of this group was very small and the correlation did not survive correction for multiple testing. Lack of associations with these two parameters is expected since TEER and E. coli uptake involve different pathways. TEER is evaluating the permeability of the duodenal epithelium to ions that can cross via the paracellular and transcellular pathway (mediated by channels and transporters). In contrast, E. coli uptake is assessing the transcellular pathway that involves endocytosis and exocytosis of bacteria. 


\section{DISCUSSION}

In this study, we aimed to further unravel the relationship between bile salts, bacterial translocation and duodenal mucosal permeability in patients with FD and HV. First, we observed a correlation between bile salts and duodenal permeability in patients with FD, in which the hydrophilic bile salt GUDCA seems to be involved. Second, GUDCA abolished the differences in duodenal epithelial resistance and bacterial passage between patients with FD and HV. Third, no correlations were found between bacterial passage and duodenal TEER in HV and patients with FD.

In the present study, a positive correlation was found between the ratio of GCA and GCDCA to TUDCA and GUDCA and TEER during fasting and fed state and also between secondary bile salts and paracellular passage during the bile salt peak in patients with FD. In agreement with our hypothesis, these data confirm a correlation between bile salts and duodenal mucosal permeability in patients with FD. Both correlations link increased GUDCA and TUDCA concentrations to increased duodenal permeability in patients with FD. However, these pilot observations of correlations did not survive correction for multiple testing, and therefore replication in a bigger cohort is warranted. Moreover, it has been suggested in the literature that UDCA conjugates play a role in maintaining the intestinal barrier integrity, which is in apparent contradiction to our data. ${ }^{19}$ However, the data from the literature were observed in mice and are based on the effect of pharmacological concentrations of exogenous UDCA rather than concentrations of the endogenous bile salts. We tested this particular ratio of GCA and GCDCA to TUDCA and GUDCA based on the alterations seen in the bile salt concentrations of patients with FD compared to HV in a previous study. ${ }^{11}$

UDCA is a hydrophilic secondary bile salt with anti-apoptotic and anti-inflammatory effects that was suggested to have therapeutic effects on the colonic intestinal barrier in inflammatory bowel disease (IBD). ${ }^{19-20}$ In addition, UDCA increases the hydrophilicity index of the circulating bile salt pool, stimulates bile secretion and protects against bile salts and cytokine toxicity. ${ }^{21}$ It has already been reported that intestinal microbiota, food and bile play a significant role in indomethacin-induced small intestinal inflammation and barrier dysfunction in rats. ${ }^{22}$ Several reports showed that oral UDCA intake 
$(10 \mathrm{mg} / \mathrm{kg}$ body weight, three or eight days of intake) decreased the severity of small intestinal injury and protects against indomethacin-induced small intestinal inflammation in rats by modulating intestinal barrier dysfunction and reducing oxidative stress. ${ }^{21-22}$ However, Uchida et al. reported that UDCA (after seven days of oral intake) worsened indomethacin-induced small intestinal inflammation in rats by increasing inflammatory scores and shortening the small intestinal length. ${ }^{23}$ Oral intake of TCDCA, on the other hand, attenuated intestinal inflammation and prevented indomethacin-induced increases in the hydrophobicity index. ${ }^{23}$

Our results and the reported effects of UDCA, UDCA conjugates and other bile salts on intestinal permeability and inflammation may differ due to the use of cancer cell lines versus normal cells, different types of tissue (human vs. rat, small intestine vs. colon), in vitro vs. in vivo, different dosage and duration of treatment and due to alterations in the in vivo bile composition. ${ }^{19}$ Golden et al. conducted multiple in vitro and in vivo studies and concluded that UDCA stimulates intestinal epithelial cell migration and protects from LPS-induced intestinal barrier damage via epithelial EGFR, COX-2, and extracellular signal-regulated kinases (ERK) activation. ${ }^{19}$ Considering the migratory and antiinflammatory effects, UDCA may be useful in the treatment of disorders involving small intestinal damage. ${ }^{19}$

The duodenal mucosal TEER, a parameter for permeability, was significantly lower in patients with FD compared to HV. These findings confirm the results from Vanheel et al. and Ishigami et al., which reported impaired duodenal barrier function in patients with FD. ${ }^{3-4}$ In our study, exposure of duodenal biopsies to GUDCA slightly altered the TEER, resulting in similar TEER values between patient and HV biopsies. This positive effect of GUDCA on duodenal TEER in patient biopsies agrees with the existing literature, showing that UDCA may stabilize membranes. ${ }^{24}$ Esteves et al. investigated the effects of the hydrophobic bile salt DCA and hydrophilic bile salt UDCA on monolayers and liposomes. ${ }^{24}$ They showed that DCA caused fluidization and rupture of monolayers and that UDCA may stabilize membranes. $^{24}$ 
Contrary to our hypothesis, decreased transmucosal bacterial passage was observed in biopsies from patients with FD compared to HV. Moreover, GUDCA exposure increased bacterial passage through patient biopsies, resulting in a similar bacterial passage as through HV biopsies, indicating the lack of a protective effect on bacterial translocation. According to the literature, bacterial translocation occurs less frequently in the small intestine compared to the large intestine. ${ }^{24-25}$ In a study by Münch et al., exposure of colonic biopsies from patients with collagenous colitis and $\mathrm{HV}$ to $100 \mu \mathrm{moh}$ CDCA or DCA in Ussing chambers resulted in an increased E. coli K12 uptake in biopsies from patients with collagenous colitis in remission. ${ }^{10}$ However, CDCA or DCA had no effect on bacterial uptake in biopsies from patients with active disease and from patients in remission due to therapy. ${ }^{10}$ In the present study, we studied the effect of 1,2 and $5 \mathrm{mM}$ of UDCA, a bile salt known for its cytoprotective effect, on duodenal - rather than colonic - biopsies in a different disorder, and all of these aspects may account for the diverging conclusions.

UDCA is available as a therapeutic agent and part of the goal of the study was to explore a potential beneficial mechanism of UDCA in patients with FD. It has been shown that bile salts modulate barrier function via EGFR or reactive oxygen species in Caco-2 cells and that nonpathogenic E. coli strains ameliorate barrier function. ${ }^{26-29}$ Münch et al. hypothesized that luminal bile salts may induce colonic inflammation in susceptible hosts due to unknown mechanisms resulting in hypersensitivity to bile salts. ${ }^{10}$ Ward et al. suggested that microbial metabolism of UDCA is necessary for the full expression of its protective effects against colonic inflammation, as they indicate that LCA, the primary metabolite of UDCA, is a more potent inhibitor of intestinal inflammatory responses than UDCA. ${ }^{30}$ Esteves et al. suggested that the exposure of monolayers to a combination of DCA and UDCA had a greater protective effect than individual bile salts. ${ }^{24}$ Based on the literature and on our own results, we hypothesize that UDCA and its conjugates may work most efficiently in vivo when combined with other bile salts and with microbial metabolization of UDCA into LCA for its full protective effect. In the present ex vivo study, exposure of duodenal tissue to high concentrations of the cytoprotective bile salt, GUDCA, elevated duodenal bacterial uptake in biopsies from susceptible hosts (patients with FD with an already impaired barrier function), while having no effect on the biopsies from HV. However, this 'negative' 
effect is limited as the bacterial uptake did not exceed the levels of biopsies from HV. The prehypothesized protective effect of GUDCA was only observed in the form of a normalized TEER.

Duodenal biopsies were exposed to 1, 2 or 5mM GUDCA for 2 hours. However, this needs to be carefully interpreted, as the sample size of the 2 and $5 \mathrm{mM}$ group is small. Furthermore, these concentrations are relatively high, as the physiological range of GUDCA in duodenal fluid of $21 \mathrm{HV}$ was between 0.04 and $0.21 \mathrm{mM}$ with an average of $0.1 \pm 0.02 \mathrm{mM} \cdot{ }^{11}$ GUDCA and TUDCA are the least abundant bile salts in human intestinal fluid. ${ }^{11}$ For more abundant bile salts, such as GCA and GCDCA, concentrations of 1 to $5 \mathrm{mM}$ can be physiological concentrations, as bile salt concentrations vary strongly inter- and intra-individually. ${ }^{11}$

Finally, there seems to be no correlation between duodenal permeability and E. coli passage in HV and in patients with FD. These results indicate that duodenal bacterial translocation is not necessarily enhanced in patients with FD and hence this may be no part of the pathophysiological mechanisms of FD, considering the limitations that we were using a duodenal and an FD unspecific, chemically killed bacterium (E. coli). It remains to be shown whether bacterial translocation in FD differs in lower parts of the gastrointestinal tract with higher bacterial exposure. Moreover, our analysis was limited to a single inactivated strain of bacteria which may not be relevant in the pathogenesis of FD. Recent emerging data on the duodenal microbiota in FD suggest that potentially disease-specific strains can be investigated. Indeed, in a small preliminary report including nine patients with FD and nine age-, genderand BMI-matched controls, the duodenal mucosal microbiota were assessed: the predominant genus was Streptococcus in both control and patient group; the anaerobic genera Prevotella, Veillonella and Actinomyces were decreased in patients with FD; the community profiles between patient and control group were significantly different. In addition, duodenal mucosal bacterial load correlated negatively with quality of life and more severe symptom responses to the standardized meal correlated positively with mucosal bacterial load. Finally, the total bacterial load correlated negatively with bacterial diversity. ${ }^{31}$ Furthermore, the intestinal microbiome alters bile salt pool composition, which may also alter the bile salt pool size. ${ }^{32}$ Gut bacteria can perform multiple biotransformations of bile salts, including the hydrolysis of conjugated bile salts by $\mathrm{BSH}$, the $7 \alpha$-dehydroxylation of CA into DCA and CDCA 
into LCA, 7ß-dehydroxylation of UDCA into LCA and oxidation and epimerization (of OH-groups at position $\mathrm{C} 3, \mathrm{C} 7$ and $\mathrm{C} 12$ ) resulting in isobile acids. ${ }^{33-34}$

This ex vivo study is limited due to the small sample size and the heterogeneous patient group. Other limitations are the fact that we only tested one specific bile salt, a single measure for permeability and one strain of bacteria, which may not be specific for the duodenum, nor for FD and is chemically killed. Also, our data were analyzed pooling the data from the different GUDCA conditions.

In summary, compared to HV, FD patients have decreased duodenal mucosal resistance which is correlated with an increased ratio of secondary over primary bile salts in duodenal aspirates. The decreased mucosal resistance is not associated with increased $E$. coli translocation across the mucosa in vitro. Exposure to the hydrophilic bile salt GUDCA abolished the differences in duodenal epithelial resistance and bacterial passage between patients with FD and HV. These reported effects identify the need for more intensive research in the effects of individual as well as combined bile salts on duodenal permeability and bacterial passage and their role in the pathophysiology of FD. For future research, it is interesting to not only use multiple bile salts in this experimental setup, but also living, duodenum and/or FD specific bacteria. 


\section{ACKNOWLEDGMENTS, FUNDING AND DISCLOSURES}

DB, RF, ÅK, TV, JT and HV designed the research study. JT and HV acquired funding. PA, JDS, HV and JT provided technical services and materials. DB, TV and JT recruited participants. DB and DR performed the research and analyzed data. DB wrote the paper.

We thank all volunteers and patients for their participation in the study. We also thank the study nurses of the Gastrointestinal Motility Unit, Leuven University Hospitals.

This work was supported by a Methusalem grant from Leuven University to Jan Tack (EZX-C9725METH/14/05). Tim Vanuytsel is a senior clinical investigator and Hanne Vanheel is a postdoctoral fellow of the Flanders Research Foundation (FWO Vlaanderen).

Competing Interests: the authors have no competing interests. 


\section{REFERENCES}

1. Stanghellini V, Chan FK, Hasler WL, Malagelada JR, Suzuki H, Tack J, et al. Gastroduodenal Disorders. Gastroenterology 2016;150(6):1380-92.

2. Vanheel H, Farre R. Changes in gastrointestinal tract function and structure in functional dyspepsia. Nat Rev Gastroenterol Hepatol 2013;10(3):142-9.

3. Vanheel H, Vicario M, Vanuytsel T, Van Oudenhove L, Martinez C, Keita AV, et al. Impaired duodenal mucosal integrity and low-grade inflammation in functional dyspepsia. Gut 2014;63(2):26271.

4. Ishigami H, Matsumura T, Kasamatsu S, Hamanaka S, Taida T, Okimoto K, et al. EndoscopyGuided Evaluation of Duodenal Mucosal Permeability in Functional Dyspepsia. Clin Transl Gastroenterol 2017;8(4):e83.

5. Camilleri M, Choi MG. Review article: irritable bowel syndrome. Aliment Pharmacol Ther 1997;11(1):3-15.

6. Erickson RA, Epsten RM Jr. Oral chenodeoxycholic acid increases small intestinal permeability to lactulose in humans. Am J Gastroenterol 1988;83(5):541-4.

7. Stenman LK, Holma R, Eggert A, Korpela R. A novel mechanism for gut barrier dysfunction by dietary fat: epithelial disruption by hydrophobic bile acids. Am J Physiol Gastrointest Liver Physiol 2013;304(3):G227-34.

8. Banerjee S, Sindberg G, Wang F, Meng J, Sharma U, Zhang L, et al. Opioid-induced gut microbial disruption and bile dysregulation leads to gut barrier compromise and sustained systemic inflammation. Mucosal Immunol 2016;9(6):1418-28.

9. Bednarska O, Walter SA, Casado-Bedmar M, Strom M, Salvo-Romero E, Vicario M, et al. Vasoactive Intestinal Polypeptide and Mast Cells Regulate Increased Passage of Colonic Bacteria in Patients With Irritable Bowel Syndrome. Gastroenterology 2017;153(4):948-60 e3.

10. Münch A, Söderholm JD, Ost A, Carlsson AH, Magnusson KE, Ström M. Low levels of bile acids increase bacterial uptake in colonic biopsies from patients with collagenous colitis in remission. Aliment Pharmacol Ther 2011;33(8):954-60. 
11. Beeckmans D, Riethorst D, Augustijns P, Vanuytsel T, Farre R, Tack J, et al. Altered duodenal bile salt concentration and receptor expression in functional dyspepsia. United European Gastroenterol $J$ 2018;6(9):1347-55.

12. Beeckmans D, Farré Marti R, Keita ÅV, Vanuytsel T, Tack JF, Vanheel H. Tu1583-The effect of bile salts on epithelial integrity and bacterial uptake in functional dyspepsia. AGA Journals Gastroenterology 2018;154(6) Supplement 1:S-957.

13. Beeckmans D, Farré R, Keita ÅV, Soderhölm JD, Tack J, Vanheel H. 005 The effect of glycoursodeoxycholic acid on epithelial integrity and bacterial uptake in duodenal biopsies of patients with functional dyspepsia and healthy volunteers. poster presentation ESNM 2017.

14. Buckley MJ, Scanlon C, McGurgan P, O'Morain CA. A validated dyspepsia symptom score. Ital J Gastroenterol Hepatol 1997;29(6):495-500.

15. Riethorst D, Mols R, Duchateau G, Tack J, Brouwers J, Augustijns P. Characterization of Human Duodenal Fluids in Fasted and Fed State Conditions. J Pharm Sci 2016;105(2):673-81.

16. Wallon C, Braaf Y, Wolving M, Olaison G, Söderholm JD. Endoscopic biopsies in Ussing chambers evaluated for studies of macromolecular permeability in the human colon. Scand $J$ Gastroenterol 2005;40(5):586-95.

17. Velin AK, Ericson AC, Braaf Y, Wallon C, Söderholm JD. Increased antigen and bacterial uptake in follicle associated epithelium induced by chronic psychological stress in rats. Gut 2004;53(4):494-500.

18. Tack J, Demedts I, Dehondt G, Caenepeel P, Fischler B, Zandecki M, et al. Clinical and pathophysiological characteristics of acute-onset functional dyspepsia. Gastroenterology 2002;122(7):1738-47.

19. Golden JM, Escobar OH, Nguyen MVL, Mallicote MU, Kavarian P, Frey MR, et al. Ursodeoxycholic acid protects against intestinal barrier breakdown by promoting enterocyte migration via EGFR- and COX-2-dependent mechanisms. Am J Physiol Gastrointest Liver Physiol 2018;315(2):G259-G271. 
20. Bernardes-Silva CF, Damiao AO, Sipahi AM, Laurindo FR, Iriya K, Lopasso FP, et al. Ursodeoxycholic acid ameliorates experimental ileitis counteracting intestinal barrier dysfunction and oxidative stress. Dig Dis Sci 2004;49(10):1569-74.

21. Poupon R. Ursodeoxycholic acid and bile-acid mimetics as therapeutic agents for cholestatic liver diseases: an overview of their mechanisms of action. Clin Res Hepatol Gastroenterol 2012;36 Suppl 1:S3-12.

22. Kullmann F, Gross V, Rüschoff J, Arndt H, Benda W, Winkler von Möhrenfels A, et al. Effect of ursodeoxycholic acid on the inflammatory activity of indomethacin-induced intestinal inflammation in rats. Z Gastroenterol 1997;35(3):171-8.

23. Uchida A, Yamada T, Hayakawa T, Hoshino M. Taurochenodeoxycholic acid ameliorates and ursodeoxycholic acid exacerbates small intestinal inflammation. Am J Physiol 1997;272(5Pt 1):G124957.

24. Esteves M, Ferreira MJ, Kozica A, Fernandes AC, Gonçalves da Silva A, Saramago B. Interaction of Cytotoxic and Cytoprotective Bile Acids with Model Membranes: Influence of the Membrane Composition. Langmuir 2015;31(32):8901-10.

25. Koh IH, Guatelli R, Montero EF, Keller R, Silva MH, Goldenberg S, et al. Where is the site of bacterial translocation--small or large bowel? Transplant Proc 1996;28(5):2661.

26. Araki Y, Katoh T, Ogawa A, Bamba S, Andoh A, Koyama S, et al. Bile acid modulates transepithelial permeability via the generation of reactive oxygen species in the Caco-2 cell line. Free Radic Biol Med 2005;39(6):769-80.

27. Raimondi F, Santoro P, Barone MV, Pappacoda S, Barretta ML, Nanayakkara M, et al. Bile acids modulate tight junction structure and barrier function of Caco-2 monolayers via EGFR activation. Am J Physiol Gastrointest Liver Physiol 2008;294(4):G906-13.

28. Zareie M, Riff J, Donato K, McKay DM, Perdue MH, Söderholm JD, et al. Novel effects of the prototype translocating Escherichia coli, strain $\mathrm{C} 25$ on intestinal epithelial structure and barrier function. Cell Microbiol 2005;7(12):1782-97.

29. Otte JM, Podolsky DK. Functional modulation of enterocytes by gram-positive and gramnegative microorganisms. Am J Physiol Gastrointest Liver Physiol 2004;286(4):G613-26. 
30. Ward JBJ, Lajczak NK, Kelly OB, O'Dwyer AM, Giddam AK, Ni Gabhann J, et al. Ursodeoxycholic acid and lithocholic acid exert anti-inflammatory actions in the colon. Am J Physiol Gastrointest Liver Physiol 2017;312(6):G550-G558.

31. Zhong L, Shanahan ER, Raj A, Koloski NA, Fletcher L, Morrison M, et al. Dyspepsia and the microbiome: time to focus on the small intestine. Gut 2017;66(6):1168-9.

32. Ridlon JM, Kang DJ, Hylemon PB, Bajaj JS. Bile acids and the gut microbiome. Curr Opin Gastroenterol 2014;30(3):332-8.

33. Ridlon JM, Harris SC, Bhowmik S, Kang DJ, Hylemon PB. Consequences of bile salt biotransformations by intestinal bacteria. Gut Microbes 2016;7(1):22-39.

34. Ridlon JM, Kang DJ, Hylemon PB. Bile salt biotransformations by human intestinal bacteria. $J$ Lipid Res 2006;47(2):241-59. 


\section{TABLES}

\begin{tabular}{|l|c|}
\hline \multicolumn{2}{|l|}{ Table 1 Demographics and clinical characteristics of the participants from study 1} \\
\hline Characteristic & Patients with functional dyspepsia \\
\hline Gender, Female:Male & $12: 6(66.7 \%$ female) \\
Age, years (range) & $35.4 .7 \pm 3.3(18-57)$ \\
Dyspeptic symptoms, DSS & $10.3 \pm 1.1$ \\
BMI, kg/m² & $23.0 \pm 0.9$ \\
Depression, PHQ-9 & $5.5(2.75-8.75)$ \\
Gastrointestinal-specific anxiety, VSI & $33.6 \pm 4.4$ \\
\hline Data are mean $\pm S E M$ or median (IQR); N=18 patients. DSS, dyspepsia symptom score; BMI, body mass index; PHQ-9, patient \\
health questionnaire 9; VSI, visceral sensitivity index.
\end{tabular}

\begin{tabular}{|c|c|c|c|c|}
\hline Symptom & absent & mild & moderate & severe \\
\hline Discomfort & 4 & 4 & 10 & 0 \\
\hline Postprandial fullness & 2 & 6 & 10 & 0 \\
\hline Bloating & 2 & 4 & 10 & 2 \\
\hline Epigastric pain & 8 & 3 & 6 & 1 \\
\hline Early satiety & 5 & 6 & 7 & 0 \\
\hline Nausea & 3 & 6 & 7 & 2 \\
\hline Vomiting & 17 & 0 & 1 & 0 \\
\hline Belching & 6 & 5 & 6 & 1 \\
\hline Epigastric burning & 4 & 10 & 3 & 1 \\
\hline
\end{tabular}




\begin{tabular}{|c|c|c|c|c|c|c|}
\hline & \multicolumn{3}{|c|}{ TEER } & \multicolumn{3}{|c|}{ Paracellular passage } \\
\hline & P-value & correlation coefficient & $\mathbf{N}$ & P-value & correlation coefficient & $\mathbf{N}$ \\
\hline \multicolumn{7}{|l|}{ Fasted state } \\
\hline Glyco-cholic acid & 0.78 & 0.07 & 17 & 0.89 & 0.04 & 15 \\
\hline Glyco-chenodeoxycholic acid & 0.87 & -0.04 & 17 & 0.99 & -0.005 & 15 \\
\hline Glyco-deoxycholic acid & 0.34 & 0.25 & 17 & 0.55 & -0.17 & 15 \\
\hline Glyco-ursodeoxycholic acid & 0.66 & -0.12 & 17 & 0.57 & -0.16 & 15 \\
\hline Tauro-cholic acid & 0.97 & -0.01 & 17 & 0.61 & 0.14 & 15 \\
\hline Tauro-chenodeoxycholic acid & 0.72 & 0.09 & 17 & 0.83 & 0.06 & 15 \\
\hline Tauro-deoxycholic acid & 0.46 & 0.19 & 17 & 0.70 & -0.11 & 15 \\
\hline Tauro-ursodeoxycholic acid & 0.40 & -0.22 & 17 & 0.59 & 0.15 & 15 \\
\hline Primary bile salts & 0.88 & 0.04 & 17 & 0.94 & 0.02 & 15 \\
\hline Secondary bile salts & 0.79 & 0.07 & 17 & 0.86 & -0.05 & 15 \\
\hline Total bile salts & 0.76 & 0.08 & 17 & 0.73 & 0.10 & 15 \\
\hline GCA+GCDCA/GUDCA+TUDCA & 0.03 & 0.56 & 15 & 0.57 & -0.17 & 13 \\
\hline \multicolumn{7}{|l|}{ Fed state } \\
\hline Glyco-cholic acid & 0.97 & -0.01 & 15 & 0.22 & 0.36 & 13 \\
\hline Glyco-chenodeoxycholic acid & 0.52 & -0.18 & 15 & 0.51 & 0.20 & 13 \\
\hline Glyco-deoxycholic acid & 0.52 & 0.18 & 15 & 0.33 & 0.30 & 13 \\
\hline Glyco-ursodeoxycholic acid & 0.19 & -0.36 & 15 & 0.97 & 0.01 & 13 \\
\hline Tauro-cholic acid & 0.39 & 0.24 & 15 & 0.66 & 0.14 & 13 \\
\hline Tauro-chenodeoxycholic acid & 0.97 & -0.01 & 15 & 0.67 & 0.13 & 13 \\
\hline Tauro-deoxycholic acid & 0.81 & 0.07 & 15 & 0.52 & 0.20 & 13 \\
\hline Tauro-ursodeoxycholic acid & 0.15 & -0.39 & 15 & 0.33 & 0.30 & 13 \\
\hline Primary bile salts & 0.48 & -0.20 & 15 & 0.28 & 0.32 & 13 \\
\hline Secondary bile salts & 0.93 & 0.03 & 15 & 0.08 & 0.50 & 13 \\
\hline Total bile salts & 0.92 & 0.03 & 15 & 0.09 & 0.49 & 13 \\
\hline GCA+GCDCA/GUDCA+TUDCA & 0.02 & 0.61 & 14 & 0.85 & -0.06 & 12 \\
\hline \multicolumn{7}{|l|}{ Bile acid peak } \\
\hline Glyco-cholic acid & 0.80 & -0.07 & 15 & 0.47 & 0.22 & 13 \\
\hline Glyco-chenodeoxycholic acid & 0.97 & -0.01 & 15 & 0.94 & -0.03 & 13 \\
\hline Glyco-deoxycholic acid & 0.89 & -0.04 & 15 & 0.14 & 0.44 & 13 \\
\hline Glyco-ursodeoxycholic acid & 0.67 & -0.12 & 15 & 0.59 & -0.17 & 13 \\
\hline Tauro-cholic acid & 0.94 & -0.02 & 15 & 0.62 & 0.15 & 13 \\
\hline Tauro-chenodeoxycholic acid & 0.77 & 0.08 & 15 & 0.70 & 0.12 & 13 \\
\hline Tauro-deoxycholic acid & 0.98 & -0.009 & 15 & 0.37 & 0.27 & 13 \\
\hline Tauro-ursodeoxycholic acid & 0.31 & -0.28 & 15 & 0.38 & 0.26 & 13 \\
\hline Primary bile salts & 0.57 & -0.16 & 15 & 0.38 & 0.27 & 13 \\
\hline Secondary bile salts & 0.60 & -0.15 & 15 & 0.03 & 0.60 & 13 \\
\hline Total bile salts & 0.44 & -0.21 & 15 & 0.15 & 0.42 & 13 \\
\hline
\end{tabular}




\begin{tabular}{|l|ccc|}
\hline \multicolumn{4}{|l|}{ Table 4 Demographics and clinical characteristics of the participants from study $\mathbf{2}$} \\
\hline Characteristic & HV & Patients with FD & P-value \\
\hline Gender, Female:Male & $15: 6(71 \%$ female) & $15: 6(71 \%$ female) & - \\
Age, years (range) & $38.8 \pm 2.9(22-61)$ & $42.7 \pm 3.2(18-60)$ & - \\
Dyspeptic symptoms, DSS & $1.0(0.0-1.5)$ & $12.0(9.3-14.0)$ & $<\mathbf{0 . 0 0 0 1}$ \\
BMI, kg/m² & $23.9 \pm 0.7$ & $23.3 \pm 0.8$ & 0.5 \\
Depression, PHQ-9 & $2.0(0.0-2.8)$ & $8.0(4.0-11.0)$ & $<\mathbf{0 . 0 0 0 1}$ \\
Gastrointestinal-specific anxiety, VSI & $2.0(0.5-5.5)$ & $33.0(22.0-45.0)$ & $<\mathbf{0 . 0 0 0 1}$ \\
\hline Data are mean $\pm S E M$ or median (IQR); N=21 for both groups. HV, healthy volunteers; FD, functional dyspepsia; DSS, dyspepsia \\
symptom score; BMI, body mass index; PHQ-9, patient health questionnaire 9; VSI, visceral sensitivity index.
\end{tabular}

\begin{tabular}{|c|c|c|c|c|}
\hline Symptom & absent & mild & moderate & severe \\
\hline Discomfort & 4 & 4 & 12 & 0 \\
\hline Postprandial fullness & 1 & 5 & 12 & 2 \\
\hline Bloating & 1 & 3 & 12 & 3 \\
\hline Epigastric pain & 5 & 4 & 11 & 0 \\
\hline Early satiety & 3 & 7 & 9 & 1 \\
\hline Nausea & 3 & 10 & 6 & 1 \\
\hline Vomiting & 15 & 4 & 1 & 0 \\
\hline Belching & 4 & 10 & 5 & 1 \\
\hline Epigastric burning & 6 & 8 & 6 & 0 \\
\hline
\end{tabular}




\section{FIGURE LEGENDS}

A

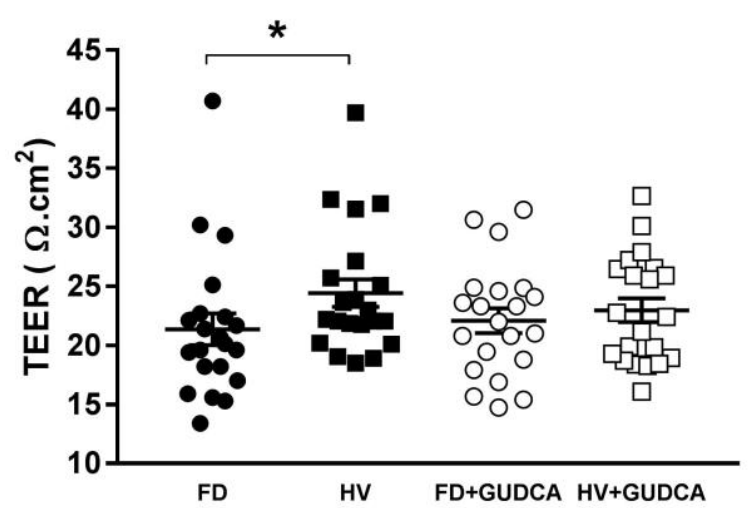

B

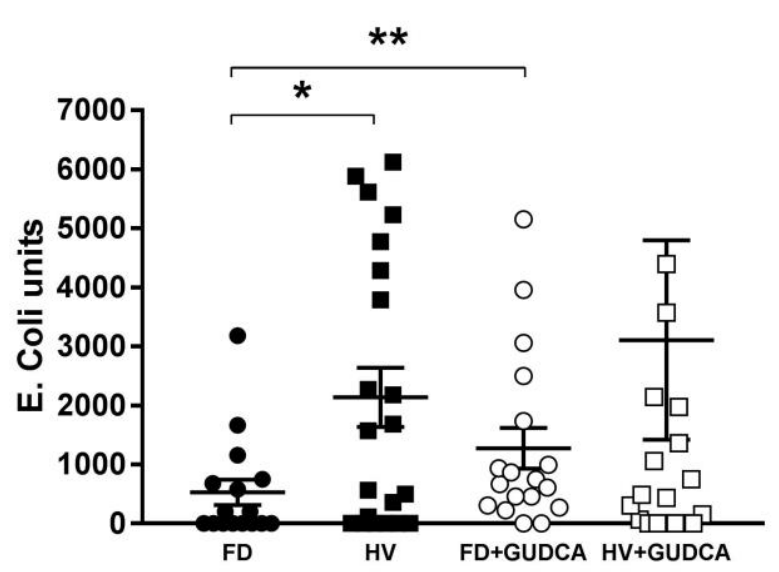

Figure 1 Glyco-ursodeoxycholic acid increased the mucosal epithelial resistance and the $E$. coli passage in duodenal biopsies of patients with functional dyspepsia. We pooled the different concentrations of GUDCA. (A) The duodenal mucosal TEER was significantly lower in biopsies from patients with FD compared to HV. After exposure to GUDCA, the TEER values between patient and $\mathrm{HV}$ biopsies were similar. (B) E. coli passage was higher in HV biopsies than in patient biopsies. Exposure to GUDCA increased $E$. coli passage in biopsies of patients with FD. $N=21$ for both groups. Data are mean $\pm S E M ;{ }^{*} p<0.05,{ }^{* *} p<0.01$. GUDCA, glyco-ursodeoxycholic acid; FD, functional dyspepsia; TEER, transepithelial electrical resistance; HV, healthy volunteers. 
A

\section{Functional dyspepsia}

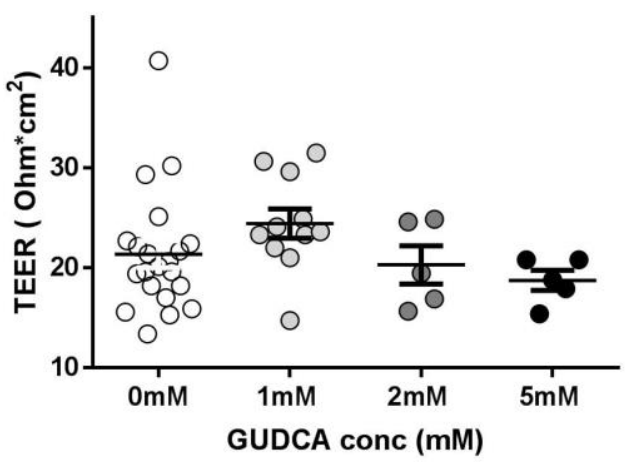

C

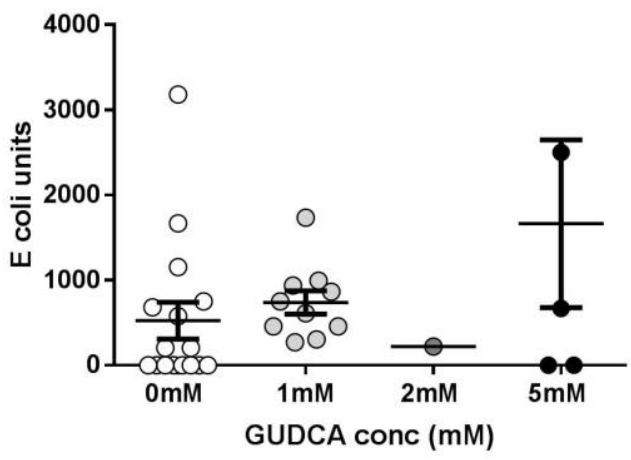

B

Healthy volunteers

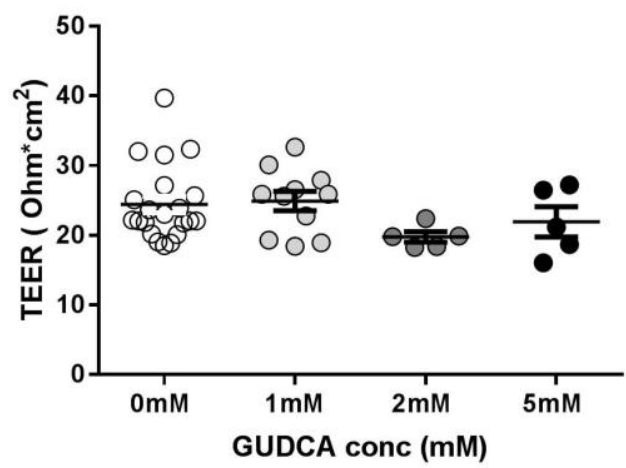

D

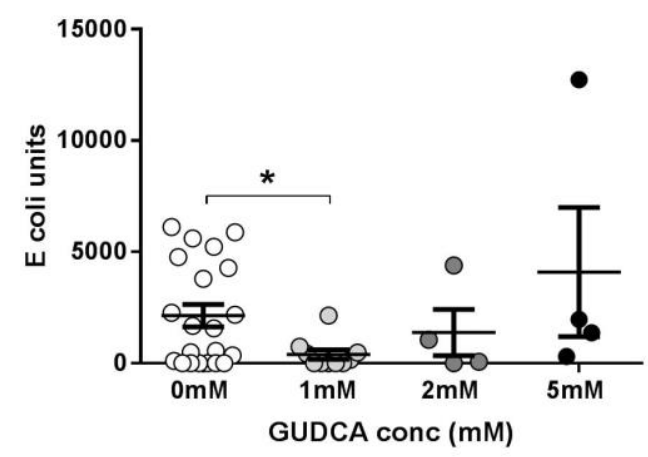

Figure 2 Exposure to $1 \mathrm{mM}$ glycoursodeoxycholic acid decreased E. coli passage in duodenal biopsies of healthy volunteers. Patient and HV duodenal biopsies were exposed to 0mM GUDCA ( $\mathrm{N}=21$ ), 1mM GUDCA ( $\mathrm{N}=11$ ), 2mM GUDCA (N=5) or 5mM GUDCA ( $N=5)$. (A-B) No effect of GUDCA on TEER in patient and HV biopsies. (C) No effect of GUDCA on E. coli passage in patient biopsies. (D) Exposure to $1 \mathrm{mM}$ GUDCA decreased E. coli passage in HV biopsies. Data are mean $\pm S E M ;{ }^{*} \mathrm{p}<0.05$. GUDCA, glycoursodeoxycholic acid; TEER, transepithelial electrical resistance. 